\title{
Análise socioeconômica e do conhecimento sobre saúde bucal das gestantes de alto
}

\section{risco}

\author{
Socioeconomic analysis and knowledge about oral health of high-risk pregnant women
}

Análisis socioeconómico y conocimiento sobre la salud bucal de las mujeres embarazadas de alto

riesgo

Recebido: 11/03/2021 | Revisado: 18/03/2021 | Aceito: 18/03/2021 | Publicado: 27/03/2021

\author{
Cláudia Batista Mélo \\ ORCID: https://orcid.org/0000-0001-5300-3510 \\ Universidade Federal da Paraíba, Brasil \\ E-mail: claudia.melo@academico.ufpb.br \\ Lucas de Medeiros Gomes \\ ORCID: https://orcid.org/0000-0003-4025-9140 \\ Universidade Federal da Paraíba, Brasil \\ E-mail: lucas.medeiros@academico.ufpb.br \\ Marcos do Nascimento Souza \\ ORCID: https://orcid.org/0000-0002-6265-2341 \\ Universidade Federal da Paraíba, Brasil \\ E-mail: marcos.souza.primeiro@gmail.com \\ Michelle Lima Alencar \\ ORCID: https://orcid.org/0000-0002-8702-7649 \\ Universidade Federal da Paraíba, Brasil \\ E-mail: michelle.alencar@academico.ufpb.br \\ Fernanda Mendes Santana \\ ORCID: https://orcid.org/0000-0002-3788-7309 \\ Universidade Federal da Paraíba, Brasil \\ E-mail: fernanda.mendes@academico.ufpb.br \\ Paulo Rogério Ferreti Bonan \\ ORCID: https://orcid.org/0000-0002-4449-4343 \\ Universidade Federal da Paraíba, Brasil \\ E-mail: pbonan@yahoo.com \\ Carmem Silvia Laureano Dalle Piagge \\ ORCID: https://orcid.org/0000-0001-7999-2943 \\ Universidade Federal da Paraíba, Brasil \\ E-mail: carmem.piagge@academico.ufpb.br
}

\begin{abstract}
Resumo
Este trabalho analisou o perfil socioeconômico e o conhecimento sobre saúde bucal das gestantes de alto risco atendidas no Hospital Universitário Lauro Wanderley (HULW) da Universidade Federal da Paraíba (UFPB). Trata-se de um estudo observacional, descritivo, transversal e de natureza quantitativa, o qual utilizou o programa Epi Info versão 7.2.4 para realizar a análise estatística dos dados. O estudo foi composto por 51 gestantes de alto risco, atendidas no HULW da UFPB, a partir da aplicação de um questionário estruturado, dividido em duas etapas; a primeira com 5 questões que abordavam o perfil socioeconômico das pacientes e a segunda com 7 questões referentes ao conhecimento e cuidados acerca da saúde bucal durante a gestação. Como parte dos resultados encontrados, em relação à faixa etária, 28 gestantes $(54,90 \%)$ possuíam entre 24 a 33 anos; $45(88,24 \%)$ possuíam até 2 saláriosmínimos como renda familiar; $51(100 \%)$ gestantes sabem o que é uma cárie, mas $33(64,7 \%)$ acreditam que a cárie não é transmitida pela saliva. Quanto ao uso de anestésicos e radiografias durante a gravidez, $31(60,78 \%)$ e 32 $(62,74 \%)$, respectivamente, das pacientes acreditam não ser possível realizar tais procedimentos durante a gravidez. Conclui-se que o conhecimento das gestantes de alto risco acerca dos cuidados em saúde bucal durante a gestação é limitado, gerando divergências e lacunas nas respostas, predominando baixa escolaridade e nível de renda.
\end{abstract}

Palavras-chave: Gravidez de alto risco; Odontologia; Saúde bucal.

\begin{abstract}
This study analyzed the socioeconomic profile and knowledge about oral health of high-risk pregnant women attended at the Lauro Wanderley University Hospital (HULW) of the Federal University of Paraíba (UFPB). This is an observational, descriptive, cross-sectional and quantitative study, which used the Epi Info program version 7.2.4 to perform statistical data analysis. The study was composed of 51 high-risk pregnant women assisted at the HULW of UFPB, from the application of a structured questionnaire, divided into two stages; the first with 5 questions that addressed the socioeconomic profile of patients and the second with 7 questions related to knowledge and care about
\end{abstract}


oral health during pregnancy. As part of the results found, in relation to age group, 28 pregnant women $(54.90 \%)$ were between 24 and 33 years old; $45(88,24 \%)$ had up to 2 minimum wages as family income; 51 (100\%) pregnant women know what caries is, but $33(64.7 \%)$ believe that caries is not transmitted by saliva. Regarding the use of anesthetics and radiographs during pregnancy, $31(60.78 \%)$ and $32(62.74 \%)$, respectively, of the patients believe that it is not possible to perform such procedures during pregnancy. It is concluded that the knowledge of high-risk pregnant women about oral health care during pregnancy is limited, generating divergences and gaps in responses, predominantly low schooling and income level.

Keywords: High risk pregnancy; Dentistry; Oral health.

\section{Resumen}

Este estudio analizó el perfil socioeconómico y el conocimiento acerca de la salud bucal de las mujeres embarazadas de alto riesgo atendidas en el Hospital Universitario Lauro Wanderley (HULW) de la Universidad Federal de Paraíba (UFPB). Se trata de un estudio observacional, descriptivo, transversal y cuantitativo, que utilizó el programa Epi Info versión 7.2.4 para realizar análisis estadísticos de datos. El estudio estuvo compuesto por 51 mujeres embarazadas de alto riesgo atendidas en el HULW de la UFPB, procedentes de la aplicación de un cuestionario estructurado, dividido en dos etapas; la primera con 5 preguntas que abordaban el perfil socioeconómico de las pacientes y la segunda con 7 preguntas relacionadas con el conocimiento y la atención sobre la salud bucal durante el embarazo. Como parte de los resultados encontrados, en relación con el grupo de edad, 28 mujeres embarazadas $(54,90 \%)$ tenían entre 24 y 33 años; $45(88,24 \%)$ tenían hasta 2 salarios mínimos como ingreso familiar; 51 (100\%) las mujeres embarazadas saben lo que es caries, pero $33(64,7 \%)$ creen que las caries no se transmiten por saliva. En cuanto al uso de anestésicos y radiografías durante el embarazo, $31(60,78 \%)$ y $32(62,74 \%)$, respectivamente, de las pacientes creen que no es posible realizar este tipo de procedimientos durante el embarazo. Se concluye que el conocimiento de las mujeres embarazadas de alto riesgo sobre la atención de salud bucal durante el embarazo es limitado, generando divergencias y lagunas en las respuestas, predominantemente bajos niveles de escolaridad e ingresos.

Palabras clave: Embarazo de alto riesgo; Odontología; Salud bucal.

\section{Introdução}

Em 2004, foram estabelecidas as diretrizes que criaram a Política Nacional de Saúde Bucal (PNSB), conhecida como "Brasil Sorridente", com o objetivo de promover, prevenir, recuperar e manter a saúde bucal dos brasileiros. A fim de superar as desigualdades sociais e estabelecer uma saúde bucal de maneira igualitária para a população, a política utilizou os princípios do Sistema Único de Saúde (SUS), constituindo-se como uma área de integralidade, que articula além dos três níveis de atenção, as ações multidisciplinares, multiprofissionais e intersetoriais (Brasil, 2018). Em áreas de baixo nível socioeconômico, para alcançar o sucesso nas medidas públicas de saúde bucal é primordial que a equipe interdisciplinar alcance as mães e as informem sobre os benefícios gerados em manter uma rotina de higiene bucal nela e nos seus filhos (Blomma \& Krevers, 2020; Jordão, Malta \& Freire, 2018).

A saúde bucal das mães é uma das grandes influenciadoras da saúde bucal infantil, porém durante a gravidez, as gestantes tendem a adiar a ida ao dentista até depois do parto (Baskaradoss \& Geevarghese, 2020). Uma das justificativas para esse fato é que na gravidez acontecem mudanças fisiológicas e psicossociais únicas no corpo de uma mulher que dificultam a gestante de procurar atendimento no serviço público de saúde. Uma das formas de transpor essa barreira e propagar conhecimento é realizar capacitação para os profissionais da saúde sobre a importância do pré-natal odontológico, desmistificando para o público-alvo os medos que estão relacionados à atenção odontológica durante esse período (Silva et al., 2020; Harb, Carmo e Boaventura, 2020; Machado et al., 2018).

Guedes-Pinto, Bönecker e Fernandes (2012) afirmam que a fonte de infecção primária dos bebês por Streptococus mutans, causador da cárie, está principalmente na saliva das mães. O veículo de transmissão geralmente é através do uso de talheres compartilhados, beijos nos lábios e qualquer outro comportamento que permita a transferência de gotículas de saliva da mãe para o bebê (Guedes-Pinto, Bönecker \& Fernandes, 2012). Ao iniciar seu pré-natal e em seu contato inicial com o ginecologista, deve haver um esclarecimento dessas questões de higiene para que sane as possíveis dúvidas sobre o assunto.

De acordo com Retori e colaboradores. (2020) a maioria das mulheres grávidas relataram escovar os dentes mais de duas vezes ao dia, no entanto, a prevalência de doenças periodontais, cárie e gengivite foi elevada e, além disso, mais da 
metade das gestantes afirmaram não visitar o dentista, o que pode influenciar na qualidade da higiene bucal. Além do inadequado cuidado em saúde bucal, outros fatores podem ocasionar tais doenças durante a gravidez, a exemplo do aumento da ingestão de alimentos ricos em açúcares devido aos desejos, mudanças hormonais (o estrogênio pode causar hipertrofia gengival e doença periodontal), bem como, o ácido presente no enjoo e vômitos matinais pode ocasionar desmineralização dental (Rodrigues, 2018; Aleixo et al., 2010; Pires et al., 2020).

A educação em saúde bucal na família é essencial para melhorar a efetividade da prevenção de doenças, uma vez que os responsáveis são fontes de melhoria na qualidade de saúde bucal das crianças e quanto mais cedo as informações no prénatal odontológico forem abordadas, melhor será a condição de saúde bucal da mãe e do futuro recém-nascido (Pomini et al., 2018). Nessa etapa da vida das mulheres é mais comum a utilização de serviços de saúde, possibilitando uma abordagem que facilita a discussão de assuntos relacionados à saúde, assim, essa gestante será uma multiplicadora de hábitos saudáveis em seu ambiente familiar, causando melhorias na saúde não só individual, mas também coletiva (Rigo, Dalazen \& Rigo Garbin, 2016).

A gestação é um fenômeno fisiológico, sendo assim, na maioria das vezes ocorre sem intercorrências, mas há casos em que as gestantes, por serem portadoras de alguma comorbidade, desenvolvem problemas que podem ser desfavoráveis tanto para o feto como para a mãe, esse grupo é chamado de "gestantes de alto risco" (Brasil, 2010). O Ministério da Saúde (2010) determina os seguintes fatores de risco gestacionais: idade maior de 35 anos, idade menor que 15 anos, baixa escolaridade e dependência de drogas lícitas e ilícitas, além disso, algumas condições clínicas pré-existentes podem provocar uma gravidez de risco, como o caso de hipertensão arterial, cardiopatias, doenças infecciosas e neoplasias (Brasil, 2010).

No contexto da saúde pública, para cuidar da saúde bucal das gestantes de alto risco, deverá ser feito o encaminhamento para Centros de Atendimentos Especializados em Odontologia, a exemplo dos Centro de Especialidades Odontológicas (CEO), com o intuito de receber o atendimento necessário e adequado. Durante a gestação há mudanças na vida da mulher, esse fato pode ser uma oportunidade para que os profissionais da saúde as orientem na aquisição de hábitos saudáveis de higiene bucal (Brasil, 2010).

Dessa forma, o objetivo deste trabalho consiste em apresentar o perfil socioeconômico, bem como, o conhecimento sobre saúde bucal das gestantes de alto riscos que buscam os serviços do ambulatório do Hospital Universitário Lauro Wanderley (HULW) da Universidade Federal da Paraíba (UFPB), além de mostrar que a saúde bucal durante a gestação é algo essencial na saúde da mulher e do bebê.

\section{Metodologia}

Foi realizado um estudo do tipo descritivo, com corte transversal, observacional com abordagem quantitativa (Rampazzo, 2002; Pereira et al., 2018), no Ambulatório Obstétrico do Hospital Universitário Lauro Wanderley da Universidade Federal da Paraíba (HULW/UFPB), em João Pessoa, Paraíba, Brasil. Os participantes do estudo foram gestantes de alto risco que buscavam atendimento no hospital, compondo uma amostra por conveniência, aleatória e não probabilística de 51 pacientes.

Inicialmente, foi definida a questão que norteou o estudo: As gestantes de alto risco têm conhecimento sobre a saúde bucal e a sua importância para a saúde do bebê? Para isso, foram utilizadas as bases de dados PUBMED/MedLine, Lilacs e Google Acadêmico empregando os seguintes descritores: gestantes, odontologia, saúde bucal. A pesquisa foi realizada mediante o preenchimento de questionário estruturado com o objetivo de identificar o perfil socioeconômico da gestante, bem como, o seu conhecimento sobre os cuidados com a sua saúde bucal durante o período de gestação.

Os dados foram coletados por meio de um questionário estruturado aplicado pelos pesquisadores, graduandos de Odontologia. Para estruturar o questionário foi feita uma revisão de literatura visando estipular o perfil correto da gestante de alto risco e seu conhecimento sobre saúde bucal na gestação. O questionário foi dividido em duas etapas, a primeira com 5 
questões sobre o perfil socioeconômico das gestantes de alto risco, incluindo as variáveis: idade, escolaridade, renda familiar, período gestacional e zona de moradia; e a segunda etapa do questionário continha 7 questões referentes sobre o conhecimento e cuidados acerca da saúde bucal durante a gestação. Para avaliação dos dados foi utilizado o programa de análise estatística de dados Epi Info versão 7.2.4.

Trata-se de uma pesquisa científica aprovada no Comitê de Ética em Pesquisa da Universidade Federal da Paraíba sob o C.A.E. de $n^{\circ}$ 67473717000005156. O estudo foi construído de acordo com as exigências da resolução №466/2012 do Conselho Nacional de Saúde. Todas as gestantes que aceitaram participar do estudo assinaram o Termo de Consentimento Livre e Esclarecido e a identidade das pacientes ficaram resguardadas.

\section{Resultados}

$\mathrm{Na}$ análise socioeconômica e epidemiológica da amostra do estudo, a maior incidência de idade das gestantes se mostrou na faixa de 24 a 33 anos com 54,9\% ( $n=28$ ), seguido de 34 a 43 anos com 29,4\% ( $n=15)$. Quase a totalidade das entrevistadas tinha renda familiar de até 2 salários mínimos em sua residência e o restante apresentava renda de 2 a 4 salários mínimos. Mais da metade das grávidas estavam no terceiro trimestre de gestação, e 3,92\% (n=2) desconhecia seu período gestacional. O grau de escolaridade apresentou maior prevalência em Ensino Médio completo e Fundamental incompleto, havendo também 6 grávidas com o Ensino Superior completo (Tabela 1). 
Tabela 1. Distribuição de dados de acordo com o perfil socioeconômico.

\begin{tabular}{|c|c|c|}
\hline Dados & $\mathbf{n}$ & $\%$ \\
\hline \multicolumn{3}{|l|}{ IDADE } \\
\hline 14 a 23 anos & 8 & 15,68 \\
\hline 24 a 33 anos & 28 & 54,90 \\
\hline 34 a 43 anos & 15 & 29,41 \\
\hline \multicolumn{3}{|l|}{ ESCOLARIDADE } \\
\hline Analfabeta & 1 & 1,96 \\
\hline Fundamental incompleto & 12 & 23,52 \\
\hline Fundamental completo & 8 & 15,68 \\
\hline Ensino Médio incompleto & 3 & 5,88 \\
\hline Ensino Médio completo & 20 & 39,21 \\
\hline Ensino Superior incompleto & 1 & 1,96 \\
\hline Ensino Superior completo & 6 & 11,76 \\
\hline \multicolumn{3}{|l|}{ RENDA FAMILIAR } \\
\hline Até 2 salários mínimos & 45 & 88,24 \\
\hline De 2 a 4 salários mínimos & 6 & 11,76 \\
\hline \multicolumn{3}{|c|}{ PERÍODO GESTACIONAL } \\
\hline $1^{\circ}$ Trimestre & 4 & 7,84 \\
\hline $2^{\circ}$ Trimestre & 15 & 29,41 \\
\hline $3^{\circ}$ Trimestre & 30 & 58,82 \\
\hline Desconhece & 2 & 3,92 \\
\hline \multicolumn{3}{|l|}{ ZONA DE MORADIA } \\
\hline Urbana & 47 & 92,16 \\
\hline Rural & 4 & 7,84 \\
\hline
\end{tabular}

Fonte: Autores.

Por estarem no período gestacional com idade acima de 35 anos, 13 (25,49\%) pacientes foram consideradas grávidas de risco, bem como, 2 (3,9\%) pacientes com idade de 14 anos.

Com base nos dados da Tabela 2, a totalidade da amostra apresentou conhecimento sobre a doença cárie, contudo 18 $(35,3 \%)$ afirmaram que é transmitida pela saliva. 30 mulheres relataram que a gestação favorece o aparecimento de lesões 
cariosas e 49 acreditam que o período gestacional acarreta problemas na saúde bucal. Indagadas sobre a possibilidade de realização de procedimentos odontológicos durante o período gestacional, 92,15\% ( $\mathrm{n}=47)$ pacientes responderam que sim. Contudo, $32(62,74 \%)$ pacientes afirmam não poder ser realizados exames radiográficos e 31 (60,78\%) relataram não poder fazer o uso de anestesias.

Tabela 2. Conhecimentos e cuidados acerca da saúde bucal durante a gestação.

\begin{tabular}{|c|c|c|}
\hline Durante a gestação & Sim & Não \\
\hline Sabe o que é cárie? & $\mathrm{n}=51(100 \%)$ & $\mathrm{n}=0(0 \%)$ \\
\hline A cárie é transmitida pela saliva? & $\mathrm{n}=18(35,3 \%)$ & $\mathrm{n}=33(64,7 \%)$ \\
\hline Fica-se favorável ao aparecimento de cáries? & $\mathrm{n}=30(58,82 \%)$ & $\mathrm{n}=21(41,18 \%)$ \\
\hline Podem acontecer mudanças que levam a problemas na saúde bucal? & $\mathrm{n}=49(96,07 \%)$ & $\mathrm{n}=2(3,93 \%)$ \\
\hline É possível realizar tratamento odontológico? & $\mathrm{n}=47(92,15 \%)$ & $\mathrm{n}=4(7,85 \%)$ \\
\hline É possível a aplicação de anestésicos? & $\mathrm{n}=20(31,37 \%)$ & $\mathrm{n}=31(60,78 \%)$ \\
\hline É possível a realização de radiografias? & $\mathrm{n}=19(29,41 \%)$ & $\mathrm{n}=32(62,74 \%)$ \\
\hline
\end{tabular}

Fonte: Autores.

\section{Discussão}

Com base nos dados obtidos, foi percebido ainda desconhecimento por parte das gestantes sobre os procedimentos odontológicos na gravidez, em que 62,74\% e 60,78\% declararam não ser possível realizar radiografia e anestesia, respectivamente. Além disso, há uma inconsistência quando a totalidade das entrevistadas assume saber o que é uma cárie, mas existe uma divisão quando se trata da questão se essa doença é transmitida ou não pela saliva. Com isso, é perceptível que muitas mulheres grávidas não são devidamente orientadas nos diversos níveis de atenção à saúde pública que tem como uma de suas premissas a promoção e prevenção de saúde.

Dados dessa pesquisa mostram que grande parte das gestantes $(58,82 \%)$ que chegaram ao terceiro trimestre de gestação ainda não possuíam orientação sobre saúde bucal. Botelho e colaboradores (2019) afirmam que "Em relação ao período ideal para realizar o tratamento odontológico de gestantes, o segundo trimestre é o momento mais indicado". O primeiro trimestre é considerado o período menos adequado, porque é quando ocorre as principais transformações embriológicas, bem como, no terceiro trimestre é prudente evitar a ida ao dentista, devido a um maior risco de hipertensão e ao desconforto na cadeira odontológica o qual pode causar hipotensão postural (Botelho et al., 2019).

Esta pesquisa mostrou o desconhecimento por parte das gestantes do agente causador da cárie e de como ocorre a propagação dessa doença, o que ressalta a necessidade de políticas públicas de promoção e prevenção da saúde bucal em gestantes de alto risco. Gislon, Bottan e Mianes (2018) investigaram a relação entre a classe social de gestantes com o seu conhecimento nos cuidados em saúde bucal na infância e concluíram que a maioria das mães pertencentes às classes sociais $\mathrm{B}$ e $\mathrm{C}$ obtiveram um nível de conhecimento médio, já as pertencentes à classe social $\mathrm{D}$ obtiveram um conhecimento baixo. $\mathrm{O}$ tópico referente às fontes de flúor, independentemente da classe social, obteve o pior índice de acertos, pois a maioria das gestantes acreditava que o dentrifício deveria estar presente em todo o comprimento da escova de dente, tal fato pode contribuir para o desenvolvimento da fluorose dentária (Gislon, Bottan \& Mianes, 2018). 
O presente estudo evidenciou a necessidade de esclarecer à população o que pode ser feito e as contra indicaçães referentes aos cuidados com a saúde bucal da gestante. Uma média de $60 \%$ das entrevistadas acreditava não poder realizar procedimentos que envolvam exposição ao raio-X. A exposição aos Raios-X deve ser evitada durante todo o período gestacional, mesmo que em doses mínimas, a exemplo de como ocorre nas radiografias odontológicas, uma vez que pode colocar em risco a integridade fetal, principalmente no primeiro trimestre (Prado et al., 2019). Na pesquisa de Prado e colaboradores (2019), 64\% dos entrevistados têm conhecimento dos riscos que a radiografia em mulheres gestantes pode ocasionar, por isso, optam por realizar o procedimento se as vantagens superarem os riscos e com o uso do avental de chumbo, principalmente protegendo a região abdominal.

A falta de informação existente entre as gestantes sobre o uso de anestésicos se faz evidente quando 60,78\% das entrevistadas acreditam que não podem fazer uso do fármaco. Os anestésicos locais são considerados seguros durante todo o período gestacional em pacientes saudáveis, mas principalmente durante o primeiro trimestre (Maragno et al., 2019). Na pesquisa de Maragno e colaboradores (2019), 42,86\% dos cirurgiões-dentistas entrevistados consideram que pode utilizar anestésicos em procedimentos odontológicos, desde que com as devidas indicações para serem usados, 28,57\% utiliza anestésicos sem vasoconstritores e 28,57\% não considera segura a utilização durante esse período.

No presente estudo, 39,21\% das gestantes possuíam o Ensino Médio Completo, seguido de 23,52\% que cursaram apenas o Ensino Fundamental Incompleto. Pacheco e colaboradores (2020) avaliaram a influência no grau de escolaridade das gestantes com a Qualidade de Vida Relacionada à Saúde Bucal (QVRSB) e concluíram que ter pouco grau de escolaridade influencia diretamente na QVRSB, uma vez que as mulheres grávidas que estudaram por mais tempo tiveram menos chance de apresentar impacto, consequentemente, uma melhor qualidade de vida.

De acordo com os resultados deste estudo, 88,24\% vivem com renda familiar de até 2 salários mínimos. Gislon, Bottan e Mianes (2018) afirmam que os diferentes níveis socioeconômicos das gestantes resultaram em lacunas significativas no conhecimento sobre itens relacionados à saúde bucal na infância (Gislon, Bottan \& Mianes, 2018).

A falta de informação das gestantes quanto à importância dos cuidados em saúde bucal é um dos motivos pelos quais elas não buscam a assistência odontológica. O pré-natal odontológico deve existir, principalmente com uma integração entre os profissionais da saúde, a fim de fornecer orientações a respeito da importância do atendimento odontológico durante a gestação (Silva et al., 2020). Por isso, é importante que os profissionais atuem na promoção e prevenção da saúde bucal durante o prénatal.

\section{Conclusão}

Conclui-se que o conhecimento das gestantes de alto risco acerca dos cuidados em saúde bucal durante a gestação é limitado, gerando divergências e lacunas nas respostas, predominando baixa escolaridade e nível de renda.

Como limitação deste estudo, observou-se a dificuldade de obter uma amostra maior, devido à especificidade do assunto pesquisado e, além disso, algumas participantes não tiveram interesse em responder o questionário.

Poderão ser realizados novos estudos correlacionando as alterações periodontais em gestantes de alto risco, como sugestão de trabalhos futuros.

\section{Referências}

Aleixo, R. Q., de Moura, C. O., de Almeida, F. A., \& Moreira, K. F. A. (2016). Alterações bucais em gestantes-revisão da literatura. Revista Saber Científico, $1(1), 68-80$.

Blomma, C., \& Krevers, B. (2020). Important aspects of conducting an interdisciplinary public preventive oral health project for children in areas with low socioeconomic status: staff perspective. BMC oral health, 20(1), 1-13. 
Baskaradoss, J. K., \& Geevarghese, A. (2020). Utilization of dental services among low and middle income pregnant, post-partum and six-month post-partum women. BMC oral health, 20, 1-9.

Brasil. Ministério da Saúde. (2018). Secretaria de Assistência à Saúde. Departamento de Atenção Básica. A Saúde Bucal no Sistema Único de Saúde. Brasília. http://bvsms.saude.gov.br/bvs/publicacoes/saude_bucal_sistema_unico_saude.pdf

Brasil. Ministério da Saúde. (2010). Secretaria de Assistência à Saúde. Gestação de alto risco. 5a ed. Brasília: Divisão Nacional de Saúde Materno-Infantil. [Série A: Normas e Manuais Técnicos] https://bvsms.saude.gov.br/bvs/publicacoes/gestacao_alto_risco.pdf

Botelho, D. L. L., Lima, V. G. A., Barros, M. M. A. F., \& de Sousa Almeida, J. R. (2019). Odontologia e gestação: a importância do pré-natal odontológico. SANARE-Revista de Políticas Públicas, 18(2).

Gislon, L. C., Bottan, E. R., \& Mianes, S. J. (2018). Saúde bucal de crianças: avaliação do conhecimento de mães de diferentes situações socioeconômicas. Clínica e Pesquisa em Odontologia-UNITAU, 9(1), 13-8.

Guedes-Pinto, A. C., Bönecker, M. J., Fernandes, F. R.C. Cárie dentária. Odontopediatria. (8a ed.), Santos, 2012:313- 345.

Harb, D. A., Carmo, W. D., Boaventura, R. M. (2020). A Importância Do Pré-Natal Odontológico. Revista Cathedral, 2(3), 145-156.

Jordão, L. M. R., Malta, D. C., \& Freire, M. D. C. M. (2018). Simultaneidade de comportamentos de risco à saúde bucal em adolescentes: evidência da Pesquisa Nacional de Saúde do Escolar. Revista Brasileira de Epidemiologia, 21, e180019.

Machado, N. E. N., Moreira, F., Gouvêa, N. S., \& Alves, F. B. T. (2018). Manual de orientações à saúde bucal da gestante durante o pré-natal odontológico. Archives of Health Investigation, 7.

Maragno, J. M., Montini, A., Rodrigues, A., Tessmann, M., \& Sonego, F. G. F. (2019). Conhecimento dos médicos e enfermeiros sobre o pré-natal odontológico em um Município da região carbonífera de Santa Catarina. Revista de Odontologia da Universidade Cidade de São Paulo, 31(1), 33-46.

Pacheco, K. T. D. S., Sakugawa, K. O., Martinelli, K. G., Esposti, C. D. D., Pacheco Filho, A. C., Garbin, C. A. S., \& Santos Neto, E. T. (2020). Saúde bucal e qualidade de vida de gestantes: a influência de fatores sociais e demográficos. Ciência \& Saúde Coletiva, 25, 2315-2324.

Pereira et al. (2018). Metodologia da pesquisa científica. UFSM.

Pires, C. C., Rodrigues, M. L., Capelli, J. D. C. S., de Souza Santos, M. M. A., \& Baião, M. R. (2020). Atenção nutricional e práticas alimentares na perspectiva de gestantes com excesso de peso. DEMETRA: Alimentação, Nutrição \& Saúde, 15, 40566.

Pomini, M. C., Galvan, J., Dias, G. F., Gouvêa, N. S. D., \& Alves, F. B. T. (2018). Prevalência de cárie em bebês e sua relação com o conhecimento e hábitos das mães. Arq. odontol, 1-9.

Prado, L., Nunes, L. M. S., Figueiredo, R. L., Silva, R. B. V., Cerdeira, C. D., \& Santos, G. B. (2019). Conduta de cirurgiões-dentistas no atendimento à paciente gestante. Revista Científica da UNIFENAS, 1(3).

Rampazzo, L. (2002). Metodologia científica para alunos de graduação e pós-graduação. Edições Loyola, 146 p.

Retori, C. P., Knorst, J. K., Bolsson, G. B., Savian, C. M., Casarin, M., \& Santos, B. Z. (2020). Associação entre a higiene bucal e qualidade de vida relacionada à saúde bucal de gestantes. Research, Society and Development, 9(1), e137911811-e137911811.

Rigo, L., Dalazen, J., Rigo, R. G. Impacto da orientação odontológica para mães durante a gestação em relação à saúde bucal dos filhos. Einstein (16794508), 14(2).

Rodrigues, F. I. (2018). Gestantes de alto risco e fatores associados à doença periodontal (Tese de Mestrado).

Silva, C. C., Maroneze, M. C., Zamberlan, C., \& dos Santos, B. Z. (2020). Capacitação sobre o pré-natal odontológico para profissionais da equipe de saúde: relato de experiência. Research, Society and Development, 9(8), e204984481-e204984481. 